\title{
Investigation of the Diagnostic Value of Ultrasonography for Radial Neuropathy Located at the Spiral Groove
}

\author{
Seojin Song, MD, Yeonji Yoo, MD, Sun Jae Won, MD, PhD, \\ Hye Jung Park, MD, Won Ihl Rhee, MD, PhD \\ Department of Rehabilitation Medicine, Yeouido St. Mary's Hospital, College of Medicine, \\ The Catholic University of Korea, Seoul, Korea
}

\begin{abstract}
Objective To determine a diagnostic cut-off value for the cross-sectional area (CSA) of the radial nerve using ultrasonography for radial neuropathy located at the spiral groove (SG).

Methods Seventeen patients with electrodiagnostic evidence of radial neuropathy at the SG and 30 healthy controls underwent ultrasonography of the radial nerve at the SG. The CSAs at the SG were compared in the patient and control groups. The CSA at the SG between the symptomatic and asymptomatic sides $(\Delta \mathrm{Sx}$-Asx and Sx/Asx, respectively) were analyzed to obtain the optimal cut-off value. The relationship between the electrophysiological severity of radial neuropathy and CSA was also evaluated.

Results Among the variables examined, there were statistically significant differences in the CSA between the patient and control groups, $\Delta \mathrm{Sx}-\mathrm{Asx}$, and Sx/Asx at the SG. In a receiver operating characteristics analysis, the cut-off CSA was $5.75 \mathrm{~mm}^{2}$ at the SG (sensitivity $52.9 \%$, specificity $90 \%$ ), $1.75 \mathrm{~mm}^{2}$ for $\Delta$ Sx-Asx (sensitivity $58.8 \%$, specificity $100 \%$ ), and $1.22 \mathrm{~mm}^{2}$ for Sx/Asx (sensitivity $70.6 \%$, specificity $93.3 \%$ ) in diagnosing radial neuropathy at the SG. There was no significant correlation between CSA and electrophysiological severity score for either patient group.

Conclusion The reference value obtained for CSA of the radial nerve at the SG may facilitate investigation of radial nerve pathologies at the SG.
\end{abstract}

Keywords Radial neuropathy, Diagnostic imaging

Received August 18, 2017; Accepted October 20, 2017

Corresponding author: Sun Jae Won

Department of Rehabilitation Medicine, Yeouido St. Mary's Hospital, College of Medicine, The Catholic University of Korea, 10, 63-ro, Yeongdeungpogu, Seoul 07345, Korea. Tel: +82-2-3779-1257, Fax: +82-2-3779-2075, E-mail: gstinfog@catholic.ac.kr

ORCID: Seojin Song (http://orcid.org/0000-0002-7956-1075); Yeonji Yoo (http://orcid.org/0000-0003-1323-4503); Sun Jae Won (http://orcid.org/00000002-9057-3747); Hye Jung Park (http://orcid.org/0000-0002-6731-4376); Won Ihl Rhee (http://orcid.org/0000-0002-4111-9978).

(c) This is an open-access article distributed under the terms of the Creative Commons Attribution Non-Commercial License (http://creativecommons.org/ licenses/by-nc/4.0) which permits unrestricted noncommercial use, distribution, and reproduction in any medium, provided the original work is properly cited. Copyright $\odot 2018$ by Korean Academy of Rehabilitation Medicine 


\section{INTRODUCTION}

The radial nerve is responsible for innervating the extensor compartments of the arm [1]. After leaving the axilla, the radial nerve travels closely around the posterolateral aspect of the humeral shaft. After distributing muscular branches to the triceps brachii and the anconeus muscles, the radial nerve wraps around the humerus in the spiral groove (SG). Because of its close proximity to the humerus and its stability when it penetrates the lateral intermuscular septum, the radial nerve becomes susceptible to compression [2]. The radial nerve is commonly injured by trauma, usually only secondary to humeral fracture, and not as commonly by external nerve compression ('Saturday night palsy') [3], related to its previously mentioned anatomical susceptibility and, rarely, by strenuous muscular effort or infarction from vasculitis [4].

The diagnosis of radial neuropathy is based on clinical history, physical examination, and electrodiagnostic study. Among these, electrodiagnosis can be used not only to evaluate the functional state of the nerve, but also to locate lesions more accurately. It is possible to demonstrate conduction blocks or decrease of conduction velocity across the lesions, using nerve conduction studies [3] or abnormal needle electromyographic findings of muscles innervated from the radial nerve $[4,5]$. To date, electrodiagnostic studies have played a key role in the diagnosis of neuropathy [6-8].

Owing to technical developments in the field of imaging techniques, ultrasonography and magnetic resonance imaging can now be used as additional tests to diagnose difficult neuropathy cases with excellent resolution and results. These techniques have unique advantages of providing the anatomical location of neuropathy and the visual information regarding etiology [9]. In particular, ultrasonography has the benefits of cost-effectiveness, real-time imaging, portability, non-invasiveness, and are compatible with implanted metal devices and are radiation-free. Consequently, they have become a widespread imaging method to evaluate the peripheral nerves $[2,10]$. Ultrasonography reveals nerve swelling and can quantify it by measurement of nerve cross-sectional area (CSA). Although measuring CSA on a transverse scan is the most commonly used examination to diagnose entrapment neuropathies [9-14], few studies have investigated cut-off values for sonographic diagnosis of radial neuropathy at the SG.

The goal of the present study is to evaluate the diagnostic value of ultrasonographic measurements, and the efficacy of ultrasonography in the diagnosis of radial neuropathy at the SG. The second goal is to assess the correlation between CSA and electrophysiological severity.

\section{MATERIALS AND METHODS}

The study protocol was approved by the Institutional Review Board of the Yeouido St. Mary's Hospital (No. SC14RISI0141). We retrospectively assessed medical records detailing the clinical and electrodiagnostic evaluation of 17 patients diagnosed with radial neuropathy at the SG and recruited 30 controls without a history of the relevant disease. All individuals in the patient and control groups were greater than 18 years of age. Patients were excluded if they had any trauma or upper arm surgery that was not related to radial neuropathy. Additionally, patients with any other neurological disease(s), such as central nervous system disease, cervical radiculopathy, brachial plexopathy, polyneuropathy, or that involving only the superficial radial nerve were excluded.

Electrodiagnosis was based on guidelines $[4,5]$ as follows: abnormal radial sensory nerve action potential (if axonal); low radial compound muscle action potential (CMAP) (if axonal); conduction block at SG (if demyelinating); and needle electromyography (EMG) abnormalities in the radial nerve innervated muscle (except the anconeus and triceps). Any drop in CMAP amplitude $\geq 50 \%$ between the proximal and distal site was defined as a conduction block. CMAP amplitude comparisons were made with the contralateral side, and reduction $\geq 50 \%$ in amplitude was defined as axonal loss [3]. For statistical analysis, a scale designed by Mondelli et al. [15] was used for electrophysiological assessment of the severity of radial neuropathy at the SG. This score was based on the following electrophysiological parameters: (1) sensory conduction velocity (SCV) and amplitude of sensory nerve action potential (SNAP); (2) percentage decrease in CMAP amplitude across the nerve lesion; (3) motor conduction velocity (MCV) across the nerve lesion; and (4) needle EMG of radial-innervated muscles (except the anconeus and triceps) (Table 1). The sampled muscles for needle EMG differed depending on the physician that 
performed the examination; there were 2-6 of the radialinnervated muscles. The brachioradialis was evaluated in all cases. The next most-sampled muscles were the extensor digitorum communis, followed by the extensor carpi radialis, and the extensor indicis proprius. The EMG score is the mean of EMG scores of radial-innervated muscles. The total electrophysiological score of radial neuropathy at the SG was the sum of all scores in each domain.

All ultrasonography examinations were performed bilaterally by a physiatrist using a 7-12 MHz linear array probe (Voluson E; GE Healthcare, Waukesha, WI, USA) that was blinded to the clinical and electrodiagnostic results of each subject. The ultrasonography examinations were performed immediately after the electrodiagnos- tic studies. All patients and controls were examined in the supine position with the forearm pronated, and the elbow moderately flexed during evaluation. The probe was carefully positioned as perpendicular as possible to the nerve, and applied with minimal pressure to avoid compression of the nerve. The radial nerve was scanned along its course from the proximal area to the SG to the antecubital fossa in the distal humerus; the largest CSA nearest the proximal area of the SG was measured at the inner border of hyperechoic epineurium of the nerve using a continuous tracing technique in both groups (Fig. 1). CSAs were measured on transverse images, and the mean values were calculated from three measurements. All captured images were re-examined by a qualified physiatrist. The following values were calculated in addition

Table 1. Electrophysiological severity scale of radial neuropathy at the spiral groove ${ }^{\text {a) }}$

\begin{tabular}{|c|c|c|c|c|}
\hline Score & SCV and amplitude of SNAP & Amplitude of CMAP ${ }^{b}$ & MCV & $\mathrm{EMG}^{\mathrm{c})}$ \\
\hline 0 & Normal & Normal $(<20 \%)$ & Normal & Normal \\
\hline 1 & $\begin{array}{l}\text { Decreased amplitude of } \\
\text { SNAP with respect to } \\
\text { unaffected side }\end{array}$ & $>20 \%,<50 \%$ & $\begin{array}{l}\text { Decreased by }<25 \% \\
\text { with respect to } \\
\text { lower limits }\end{array}$ & $\begin{array}{l}\text { Reduced interferential pat- } \\
\text { tern at full effort with or } \\
\text { without denervation } \\
\text { activity at rest }\end{array}$ \\
\hline 2 & $\begin{array}{l}\text { Absolutely decreased } \\
\text { amplitude of SNAP, } \\
\text { normal SCV }\end{array}$ & $>50 \%,<100 \%$ & $\begin{array}{l}\text { Decreased by }>25 \% \\
\text { with respect to } \\
\text { lower limits }\end{array}$ & $\begin{array}{l}\text { Discrete interferential } \\
\text { pattern at full effort }\end{array}$ \\
\hline 3 & $\begin{array}{l}\text { Decreased SCV and } \\
\text { amplitude of SNAP }\end{array}$ & Absence of CMAP & Absence of CMAP & $\begin{array}{l}\text { Single interferential pattern } \\
\text { at full effort }\end{array}$ \\
\hline 4 & Absence of SNAP & & & No MUAP \\
\hline
\end{tabular}

SCV, sensory conduction velocity; SNAP, sensory nerve action potential; CMAP, compound muscle action potential; MCV, motor conduction velocity; EMG, electromyography; MUAP, motor unit action potential.

${ }^{\text {a) }}$ Scale from Mondelli et al. [15].

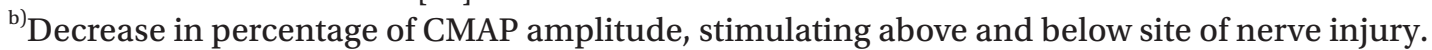

c) The EMG score is the mean of EMG scores of affected radial-innervated muscles (except, the anconeus and triceps).
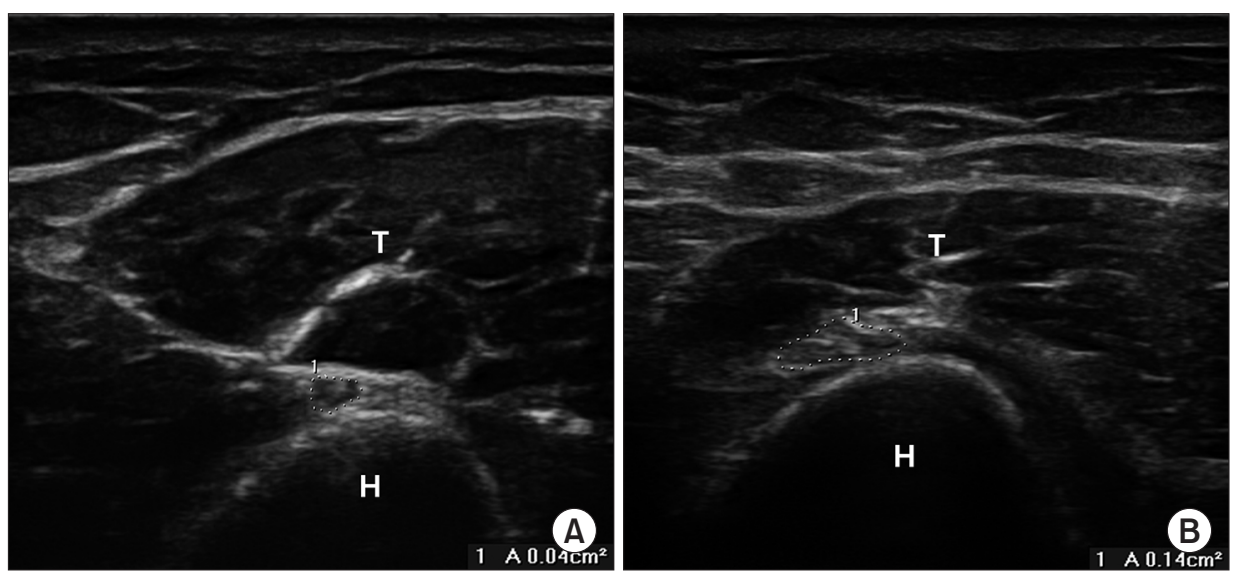

Fig. 1. Ultrasonographic images of the radial nerve at a site just proximal to the spiral groove. The radial nerve is shown inside the dotted line. (A) Radial nerve in normal controls and (B) radial nerve in patients. $\mathrm{H}$, humerus. T, triceps. 
to CSA: differences in CSA at the SG between symptomatic and asymptomatic sides ( $\triangle \mathrm{Sx}$-Asx); ratio of CSA at the SG between symptomatic and asymptomatic sides (Sx/ Asx). In the control group, differences and ratio of CSA at the SG were obtained between the right and left sides.

\section{Statistical analyses}

Statistical analysis was performed using SPSS version 21.0 (IBM, Armonk, NY, USA) for Windows (Microsoft Corporation, Redmond, WA, USA). The chi-square, t-test, and Mann-Whitney test were used to compare variables in the patient and control groups. The normality of the data was verified using the Shapiro-Wilk test. The MannWhitney test was used to analyze differences in CSA measurements between the two groups; $p<0.05$ was considered statistically significant in all tests. Receiver operating characteristic (ROC) curve analysis was performed to determine optimal cut-off points of the measurements using ultrasonography in diagnosing radial neuropathy at the SG. Spearman correlation coefficient was used to examine the relationships between the values of CSA and electrophysiological severity scale scores.

\section{RESULTS}

In this study, all 17 patients and 30 healthy controls

Table 2. Comparison of demographic characteristics of patients and controls

\begin{tabular}{lccc}
\hline \multicolumn{1}{c}{ Characteristic } & $\begin{array}{c}\text { Controls } \\
(\mathbf{n}=\mathbf{3 0})\end{array}$ & $\begin{array}{c}\text { Patients } \\
(\mathbf{n}=\mathbf{1 7})\end{array}$ & p-value \\
\hline Sex & & & 0.29 \\
$\quad$ Male & 15 & 12 & \\
$\quad$ Female & 15 & 5 & \\
Age (yr) & $45.23 \pm 13.58$ & $43.24 \pm 16.49$ & 0.67 \\
$\begin{array}{l}\text { Duration of } \\
\text { symptoms (mo) }\end{array}$ & - & $3(0.86-17)$ & \\
Height $(\mathrm{cm})$ & $165.23 \pm 7.24$ & $171.00 \pm 9.68$ & 0.07 \\
Weight $(\mathrm{kg})$ & $63.62 \pm 10.84$ & $69.62 \pm 11.18$ & 0.13 \\
\hline BMI $\left(\mathrm{kg} / \mathrm{m}^{2}\right)$ & $23.24 \pm 3.24$ & $23.72 \pm 2.59$ & 0.61 \\
\hline Side & & & \\
\hline Right & 30 & 10 & \\
\hline Left & 0 & 7 & \\
\hline
\end{tabular}

Values are presented as mean \pm standard deviation or median (1st quartile-3rd quartile).

BMI, body mass index. underwent clinical examination, and electrodiagnostic and ultrasonographic evaluations of the radial nerve. No significant differences in baseline characteristics between the patients and controls were observed ( $p>0.05)$ (Table 2). The duration between the onset of symptom(s) and the time of clinic visit ranged from one week to 43 months (median, 3 months).

Humerus fractures $(n=10)$ were the most common cause of radial neuropathies, followed by external compressions $(\mathrm{n}=7)$.

The electrophysiological severity scale scores are reported in Table 3. Except for the EMG score, there was a statistical difference in electrophysiological severity scale

Table 3. Electrophysiological severity scale scores in patients with radial neuropathy at the spiral groove

\begin{tabular}{lccc}
\hline \multicolumn{1}{c}{ Parameter } & $\begin{array}{c}\text { Humerus } \\
\text { fracture } \\
(\mathbf{n}=\mathbf{1 0})\end{array}$ & $\begin{array}{c}\text { External } \\
\text { compression } \\
(\mathbf{n}=7)\end{array}$ & Total \\
\hline $\begin{array}{c}\text { SCV and ampli- } \\
\text { tude of SNAP }\end{array}$ & $2.50 \pm 1.08$ & $0^{* *}$ & $1.47 \pm 1.50$ \\
$\begin{array}{c}\text { Amplitude of } \\
\text { CMAP }\end{array}$ & $2.70 \pm 0.48$ & $1.43 \pm 0.53^{* *}$ & $2.18 \pm 0.81$ \\
MCV & $2.50 \pm 0.97$ & $0.14 \pm 0.38^{* *}$ & $1.53 \pm 1.42$ \\
EMG & $2.29 \pm 1.27$ & $1.60 \pm 1.00$ & $2.00 \pm 1.18$ \\
Total & $9.99 \pm 2.90$ & $3.17 \pm 1.18^{* *}$ & $7.18 \pm 4.15$ \\
\hline
\end{tabular}

Values are presented as mean \pm standard deviation.

SCV, sensory conduction velocity; SNAP, sensory nerve action potential; CMAP, compound muscle action potential; MCV, motor conduction velocity; EMG, electromyography.

${ }^{* *} \mathrm{p}<0.01$, differences between each group separated according to etiology.

Table 4. Ultrasonographic measurements of the radial nerve of the subjects

\begin{tabular}{lccc}
\hline Characteristic & Controls & Patients & p-value \\
\hline $\begin{array}{c}\text { CSA at the SG } \\
\left(\mathrm{mm}^{2}\right)\end{array}$ & $4.65 \pm 0.82$ & $7.01 \pm 2.97$ & $0.005^{* *}$ \\
$\begin{array}{c}\Delta \text { Sx-Asx at the SG } \\
\left(\mathrm{mm}^{2}\right)\end{array}$ & $0.07 \pm 0.58$ & $2.54 \pm 2.45$ & $<0.001^{* *}$ \\
\hline Sx/Asx at the SG & $1.02 \pm 0.14$ & $1.62 \pm 0.66$ & $<0.001^{* *}$ \\
\hline
\end{tabular}

Values are presented as mean \pm standard deviation. CSA, cross-sectional area; SG, spiral groove; $\Delta$ Sx-Asx, differences of CSA at the SG between symptomatic and asymptomatic sides; Sx/Asx, ratio of CSA at the SG between symptomatic and asymptomatic sides.

${ }^{* *} \mathrm{p}<0.01$. 
Table 5. Cut-off values for diagnosing radial neuropathy at the spiral groove, with sensitivity and specificity

\begin{tabular}{|c|c|c|c|c|}
\hline Parameter & Cut-off & $\begin{array}{l}\text { Sensitiv- } \\
\text { ity (\%) }\end{array}$ & $\begin{array}{l}\text { Specific- } \\
\text { ity }(\%)\end{array}$ & AUC \\
\hline $\begin{array}{l}\text { CSA at the SG } \\
\left(\mathrm{mm}^{2}\right)\end{array}$ & 5.75 & 52.9 & 90.0 & 0.748 \\
\hline $\begin{array}{l}\begin{array}{l}\Delta \text { Sx-Asx at the SG } \\
\left(\mathrm{mm}^{2}\right)\end{array}\end{array}$ & 1.75 & 58.8 & 100 & 0.858 \\
\hline Sx/Asx at the SG & 1.22 & 70.6 & 93.3 & 0.854 \\
\hline
\end{tabular}

CSA, cross-sectional area; SG, spiral groove, $\Delta$ Sx-Asx, differences of CSA at the SG between symptomatic and asymptomatic sides; Sx/Asx, ratio of CSA at the SG between symptomatic and asymptomatic sides; AUC, area under the curve.

between each patient group divided according to etiology.

Table 4 summarizes CSA values at the $\mathrm{SG}$, the $\Delta \mathrm{Sx}-$ Asx, and Sx/Asx between the patient and control groups. In the patient group, the mean CSA of the radial nerve at the SG was $7.01 \pm 2.97 \mathrm{~mm}^{2}$, which was significantly larger than the corresponding area in the control group $(\mathrm{p}<0.01)$. In addition, the $\Delta \mathrm{Sx}-\mathrm{Asx}$ and $\mathrm{Sx} / \mathrm{Asx}$ were larger than that of the control group ( $\mathrm{p}<0.001$ for all).

ROC curve analysis was used to assess cut-off points for ultrasonographic measurements, and its diagnostic sensitivity and specificity. The cut-off values for diagnosis of radial neuropathy at the SG with relatively high sensitivity and specificity were $5.75 \mathrm{~mm}^{2}$ (sensitivity $52.9 \%$, specificity $90 \%$ ) for radial nerve CSA at the SG, $1.75 \mathrm{~mm}^{2}$ for $\Delta$ Sx-Asx (sensitivity $58.8 \%$, specificity $100 \%$ ), and 1.22 for Sx/Asx (sensitivity 70.6\%, specificity 93.3\%) (Table 5).

No significant correlations with radial nerve CSA at the SG and the electrophysiological severity scale were observed ( $p>0.05$ ) in all patients and subgroups divided according to etiology. Additionally, there were no significant differences in radial nerve CSA at the SG between the humerus fracture and external compression group ( $p>0.05)$.

\section{DISCUSSION}

The aim of this study was to assess the diagnostic efficacy of ultrasonography in addition to electrodiagnostic study by measuring the CSA of the radial nerve at the SG. Ultrasonography appeared to be an adequately sensitive and specific test in revealing radial nerve swelling at the SG. At the SG, patients exhibited a significantly larger mean radial nerve CSA than healthy controls, which was also reported in a previous study [3]. We found several cut-off values that were statistically significant in diagnosing radial neuropathy at the SG. This was the first study to research and propose diagnostic cut-off values for ultrasonography in radial neuropathy at the SG.

There have been many studies supporting the usefulness of ultrasonography as an adjunctive diagnostic tool to electrodiagnostic studies for peripheral neuropathy. However, most of these studies investigated the median and ulnar nerves $[9,11,12,16,17]$, and seldom used ultrasonography to diagnose radial neuropathy. That is, except for several studies describing trauma or cyst(s) that examined the causes of radial neuropathy $[7,18-20]$.

For the radial nerve site we evaluated, normal reference values have been previously reported [10,21-23]. Although some of these studies used the same nerve tracing method, there were few discrepancies in the suggested normal values. Potential explanations for these differences are that the participants differed in ethnicity, gender, age, height, and weight distribution. The different frequency of transducers used or measurement error may be other possible causes. To minimize these differences, future studies should compare the results of this study with investigations that used similar conditions. In one previous study [21], the normal reference value for the CSA of the radial nerve was $4.61 \pm 0.88 \mathrm{~mm}^{2}$. Based on this normal value, despite the relatively low sensitivity, the cut-off value of $5.75 \mathrm{~mm}^{2}$ (sensitivity $52.9 \%$, specificity $90 \%$ ) determined in our study appears to be appropriate to diagnose radial neuropathy at the SG (Table 5). For higher sensitivity, we propose $4.95 \mathrm{~mm}^{2}$ (sensitivity $82.4 \%$, specificity $60 \%$ ) as an alternative cut-off value. This value overlaps with the aforementioned normal reference; therefore, it is likely to include many false positives. To overcome this weakness, it would be helpful to verify additional $\Delta \mathrm{Sx}-\mathrm{Asx}$ and $\mathrm{Sx} /$ Asx values for diagnoses in patients with the suspected disease.

As mentioned earlier, in many other studies investigating CSA reference values for the sonography of the peripheral nerves, the CSA appeared to be correlated with gender [24], height [21], weight, body mass index, and age $[23,25]$. Therefore, using the contralateral side as an internal control $[26,27]$ to verify $\Delta S x-A s x$ and $S x / A s x$, we can reduce these biases. The mean value of $\Delta \mathrm{Sx}$-Asx was $2.54 \pm 2.45 \mathrm{~mm}^{2}$, and $1.75 \mathrm{~mm}^{2}$ was the cut-off value of 
$\Delta \mathrm{Sx}$-Asx in radial neuropathy at the SG, with a sensitivity of $58.8 \%$ and specificity of $100 \%$. The mean value of the $\mathrm{Sx} /$ Asx ratio was $1.62 \pm 0.66$, and the cut-off value for radial neuropathy at the SG was 1.22 (sensitivity of $70.6 \%$, specificity of 93.3\%). Although $\Delta \mathrm{Sx}$-Asx and Sx/Asx were useful, they are not applicable to bilateral or diffused lesions $[26,28]$ For these cases, electrodiagnostic study is predicted to play a better role.

In this study, the only characteristic that had a statistically significantly larger CSA was at the SG. The CSA values in the antecubital fossa, which we did not examine in this study, may also be larger in some cases. In an ultrasonography study investigating ulnar neuropathy at the elbow [29], the CSA was significantly larger, not only at the medial epicondyle, but also distally, farthest from the medial epicondyle, and even at the wrist. In another study [30], significantly larger CSAs were also noted $5 \mathrm{~cm}$ proximal from and distal to the medial epicondyle. Collectively, these findings could explain that the pattern of edema that occurs after nerve injury may differ depending on the site, degree, and mechanism of injury. Therefore, if the maximal swelling point was found in the nonSG portion, additional $\Delta \mathrm{Sx}$-Asx or Sx/Asx inquiry can be used to supplement the diagnosis.

In addition to determining the diagnostic value of ultrasonographic measurements in radial neuropathy at the SG, we performed a correlation analysis of CSAs and electrophysiological severity scale scores and found no significant correlation. Although not related to radial neuropathy, the relationship between ultrasonographic measurements and electrodiagnostic studies has been reported in several previous studies [16,29,31-33], but there have been conflicting results. This could be explained by several factors such as variability in nerve pathologies related to causes, relationship(s) with peripheral tissue, and the concept that ultrasound primarily assesses anatomy and electrodiagnostic studies primarily assess nerve function [16].

There were some limitations to this study. First, because of the low incidence of radial neuropathy compared with other nerve lesions, this study had a relatively small number of subjects; therefore, it may be difficult to generalize the results. Second, there was variability in the duration between the onset of symptoms and the time to the clinic visit. This could have altered the degree of nerve swelling and possibly influenced the outcomes.
Third, the cut-off value of radial CSA at the SG in the present study had a relatively lower sensitivity than that reported in other studies investigating other neuropathies. This may be because we only recruited patients with electrophysiologically confirmed radial neuropathy at the SG, and used fixed points of measurement of CSA at the SG to achieve this high level of standardization in our results. Fourth, intra-rater and inter-rater reliability were not evaluated. However, for intra-rater agreement, we calculated the mean CSA from three measurements. For inter-rater agreement, an experienced and qualified physiatrist reviewed the ultrasonographic images. Despite these shortcomings, this investigation is the first original study to propose diagnostic ultrasonography cutoff values for radial neuropathy at the SG.

In conclusion, ultrasonography is a useful additional tool to electrodiagnosis for diagnosing radial neuropathy at the SG. Our study may be helpful for the diagnosis of radial neuropathy at the SG in clinical practice. Further studies are required to confirm our findings on a larger scale and in different ethnic groups.

\section{CONFLICT OF INTEREST}

No potential conflict of interest relevant to this article was reported.

\section{REFERENCES}

1. Wang LH, Weiss MD. Anatomical, clinical, and electrodiagnostic features of radial neuropathies. Phys Med Rehabil Clin N Am 2013;24:33-47.

2. Kara M, Ozcakar L, De Muynck M, Tok F, Vanderstraeten G. Musculoskeletal ultrasound for peripheral nerve lesions. Eur J Phys Rehabil Med 2012;48:665-74.

3. Lo YL, Fook-Chong S, Leoh TH, Dan YF, Tan YE, Lee MP, et al. Rapid ultrasonographic diagnosis of radial entrapment neuropathy at the spiral groove. J Neurol Sci 2008;271:75-9.

4. Preston DC, Shapiro BE. Electromyography and neuromuscular disorders: clinical-electrophysiological correlation. 3rd ed. London: Elsevier/Saunders; 2013. p. 336-40.

5. Dumitru D, Amato AA, Zwarts MJ. Electrodiagnostic medicine. 2nd ed. Philadelphia: Hanley \& Belfus; 2002. p. 1089-90. 
6. Girtler MT, Krasinski A, Dejaco C, Kitzler HH, Cui LG, Sherebrin S, et al. Feasibility of 3D ultrasound to evaluate upper extremity nerves. Ultraschall Med 2013;34: 382-7.

7. Ong C, Nallamshetty HS, Nazarian LN, Rekant MS, Mandel S. Sonographic diagnosis of posterior interosseous nerve entrapment syndrome. Radiol Case Rep 2016;2:1-4.

8. Yalcin E, Akyuz M, Onder B. Early radial digital neuropathy of the thumb due to flexor pollicis longus tendinitis: value of ultrasound in an uncommon mild neuropathy. Muscle Nerve 2013;47:772-5.

9. Kutlar N, Bayrak AO, Bayrak İK, Canbaz S, Turker H. Diagnosing carpal tunnel syndrome with Doppler ultrasonography: a comparison of ultrasonographic measurements and electrophysiological severity. Neurol Res 2017;39:126-32.

10. Cartwright MS, Passmore LV, Yoon JS, Brown ME, Caress JB, Walker FO. Cross-sectional area reference values for nerve ultrasonography. Muscle Nerve 2008;37: 566-71.

11. Chiou HJ, Chou YH, Cheng SP, Hsu CC, Chan RC, Tiu CM, et al. Cubital tunnel syndrome: diagnosis by high-resolution ultrasonography. J Ultrasound Med 1998;17:643-8.

12. Beekman R, Visser LH. High-resolution sonography of the peripheral nervous system: a review of the literature. Eur J Neurol 2004;11:305-14.

13. Visser LH, Hens V, Soethout M, De Deugd-Maria V, Pijnenburg J, Brekelmans GJ. Diagnostic value of highresolution sonography in common fibular neuropathy at the fibular head. Muscle Nerve 2013;48:171-8.

14. Kerasnoudis A, Tsivgoulis G. Nerve ultrasound in peripheral neuropathies: a review. J Neuroimaging 2015; 25:528-38.

15. Mondelli M, Morana P, Ballerini M, Rossi S, Giannini F. Mononeuropathies of the radial nerve: clinical and neurographic findings in 91 consecutive cases. J Electromyogr Kinesiol 2005;15:377-83.

16. van Veen KE, Wesstein M, van Kasteel V. Ultrasonography and electrodiagnostic studies in ulnar neuropathy: an examination of the sensitivity and specificity and the correlations between both diagnostic tools. J Clin Neurophysiol 2015;32:240-3.

17. Nakamichi K, Tachibana S. Ultrasonographic measurement of median nerve cross-sectional area in idiopathic carpal tunnel syndrome: diagnostic accuracy. Muscle Nerve 2002;26:798-803.

18. Cartwright MS, Yoon JS, Lee KH, Deal N, Walker FO. Diagnostic ultrasound for traumatic radial neuropathy. Am J Phys Med Rehabil 2011;90:342-3.

19. Qi HT, Wang XM, Li SY, Wang GB, Wang DH, Wang ZT, et al. The role of ultrasonography and MRI in patients with non-traumatic nerve fascicle torsion of the upper extremity. Clin Radiol 2013;68:e479-83.

20. Choi SY, Park JW, Kim DH. Ultrasonographic and surgical findings of acute radial neuropathy following blunt trauma. Am J Phys Med Rehabil 2016;95:e177e182.

21. Won SJ, Kim BJ, Park KS, Yoon JS, Choi H. Reference values for nerve ultrasonography in the upper extremity. Muscle Nerve 2013;47:864-71.

22. Chen J, Wu S, Ren J. Ultrasonographic reference values for assessing normal radial nerve ultrasonography in the normal population. Neural Regen Res 2014;9: 1844-9.

23. Qrimli M, Ebadi H, Breiner A, Siddiqui H, Alabdali M, Abraham A, et al. Reference values for ultrasonograpy of peripheral nerves. Muscle Nerve 2016;53:538-44.

24. Boehm J, Scheidl E, Bereczki D, Schelle T, Aranyi Z. High-resolution ultrasonography of peripheral nerves: measurements on 14 nerve segments in 56 healthy subjects and reliability assessments. Ultraschall Med 2014;35:459-67.

25. Kerasnoudis A, Pitarokoili K, Behrendt V, Gold R, Yoon MS. Cross sectional area reference values for sonography of peripheral nerves and brachial plexus. Clin Neurophysiol 2013;124:1881-8.

26. Tagliafico A, Cadoni A, Fisci E, Bignotti B, Padua L, Martinoli C. Reliability of side-to-side ultrasound cross-sectional area measurements of lower extremity nerves in healthy subjects. Muscle Nerve 2012;46:71722.

27. Tagliafico A, Martinoli C. Reliability of side-to-side sonographic cross-sectional area measurements of upper extremity nerves in healthy volunteers. J Ultrasound Med 2013;32:457-62.

28. Yalcin E, Unlu E, Akyuz M, Karaahmet OZ. Ultrasound diagnosis of ulnar neuropathy: comparison of symptomatic and asymptomatic nerve thickness. J Hand Surg Eur Vol 2014;39:167-71.

29. Bayrak AO, Bayrak IK, Turker H, Elmali M, Nural MS. 
Ultrasonography in patients with ulnar neuropathy at the elbow: comparison of cross-sectional area and swelling ratio with electrophysiological severity. Muscle Nerve 2010;41:661-6.

30. Ellegaard HR, Fuglsang-Frederiksen A, Hess A, Johnsen B, Qerama E. High-resolution ultrasound in ulnar neuropathy at the elbow: a prospective study. Muscle Nerve 2015;52:759-66.

31. Mondelli M, Filippou G, Frediani B, Aretini A. Ultrasonography in ulnar neuropathy at the elbow: relationships to clinical and electrophysiological findings.
Neurophysiol Clin 2008;38:217-26.

32. Kim MK, Jeon HJ, Park SH, Park DS, Nam HS. Value of ultrasonography in the diagnosis of carpal tunnel syndrome: correlation with electrophysiological abnormalities and clinical severity. J Korean Neurosurg Soc 2014;55:78-82.

33. Zyluk A, Walaszek I, Szlosser Z. No correlation between sonographic and electrophysiological parameters in carpal tunnel syndrome. J Hand Surg Eur Vol 2014;39:161-6. 